\title{
Sismicidade na Zona Sísmica Acaraú, região nordeste do Brasil, entre 2008 e 2018
}

\author{
Paulo Henrique Sousa de Oliveira ${ }^{1}$, Joaquim Mendes Ferreira ${ }^{2}$, Francisco Hilário Rego Bezerra ${ }^{2}$, Aderson Farias do \\ Nascimento ${ }^{2}$ \\ 1-Escola de Ciências e Tecnologia, Universidade Federal do Rio Grande do Norte. \\ 2-Programa de Pós-Graduação em Geodinâmica e Geofísica/Universidade Federal do Rio Grande do Norte.
}

Copyright 2018, SBGf - Sociedade Brasileira de Geofísica

Este texto foi preparado para a apresentação no VIII Simpósio Brasileiro de Geofísica, Salinópolis, 18 a 20 de setembro de 2018. Seu conteúdo foi revisado pelo Comitê Técnico do VIII SimBGf mas não necessariamente representa a opinião da SBGf ou de seus associados. É proibida a reprodução total ou parcial deste material para propósitos comerciais sem prévia autorização da SBGf.

\section{Resumo}

A Zona Sísmica Acaraú (ZSA) está localizada na margem noroeste da Província Borborema. Com relatos de eventos ocorridos no século XIX e a numerosa, intensa e recente sismicidade nos últimos 30 anos (1988-2018), a ZSA tornou-se uma das principais regiões com sismicidade ativa no Brasil. Em 2008, uma intensa atividade sísmica abalou a população de Sobral com um evento atingindo $4,2 \mathrm{~m}_{\mathrm{b}}$. Em 2009, foi registrada outra grande atividade em Santana do Acaraú $(54 \mathrm{~km}$ de Sobral). De 2015 até o momento, a cidade de Irauçuba (70 km de Sobral), vem sendo fortemente abalada. Com as redes sismográficas locais instaladas nestes locais, foi possível observar que a sismicidade na ZSA vem crescendo de forma intensa e numerosa nos últimos 10 anos e que não apresenta correlação com o lineamento transbrasiliano, mas que em alguns locais, está correlacionada com estruturas locais em superfície.

\section{Introdução}

A ZSA - Zona Sísmica Acaraú (Oliveira et al., 2015) é uma das principais áreas sísmicas ativas do Brasil e possui sismicidade intraplaca desde o século XIX (Ferreira \& Assumpção, 1983), como mostrado na Figura 01. Além disso, está localizada na Província Borborema entre dois domínios geotectônicos: o Domínio Médio Coreáu e o Domínio Ceará Central (Santos et al. 2008). Esses domínios possuem diversas unidades litoestratigráficas e várias zonas de cisalhamento com trend NE-SW, destacando-se o LT - Lineamento Transbrasiliano (Figura 02), uma extensa faixa milonítica que se estende para nordeste, até a África Ocidental (falha de Kandi), e para sudoeste, até a região central do Brasil (Caby \& Arthaud 1986).

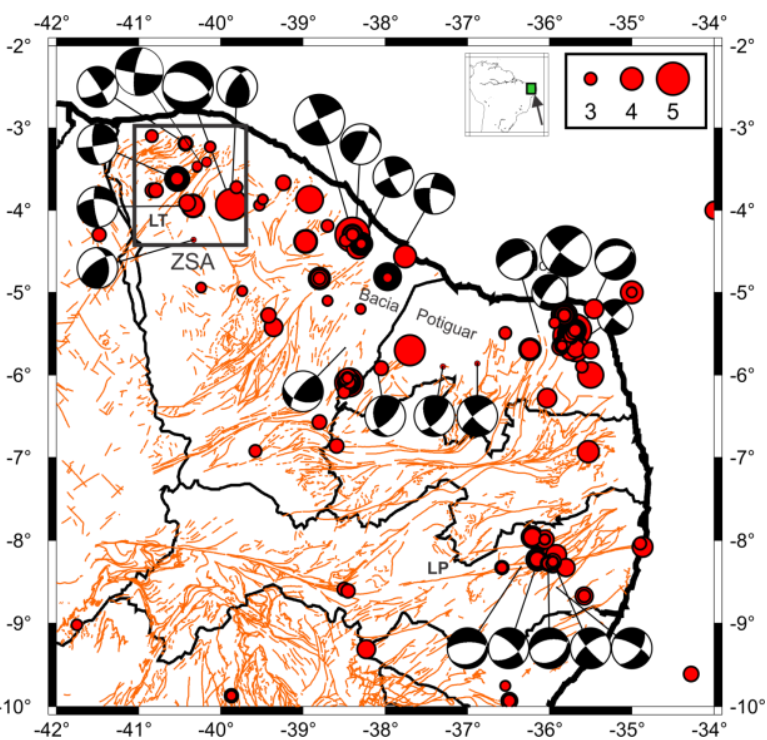

Figura 01 - Sismicidade na Província Borborema entre 1720 e 2013 com magnitudes acima de 2,5 mostrando a Zona Sísmica Acaraú (quadrado na parte superior esquerda). As "bolas de praia" representam mecanismos focais. $L T$ e $L P$ representam os Lineamentos Transbrasiliano e Pernambuco, respectivamente. Os traços laranjas são falhas mapeadas (CPRM, 2009) e o quadrado indica a área de estudo. Fonte: Boletim Sísmico Brasileiro (USP, UFRN, UnB, IPT, UNESP).

Neste trabalho é mostrado como a sismicidade na ZSA vem ocorrendo nos últimos 10 anos através das redes sismográficas com estações digitais instaladas em três locais. Local 1: Serra da Meruoca, Sobral; Local 2: Santana do Acaraú e Local 3: Irauçuba (Menezes, 2017). Os melhores sismos foram selecionados para calcular parâmetros hipocentrais e de falhamento, possiblitando analisar como vem ocorrendo a sismicidade e possível correlação com feições geológicas locais. 


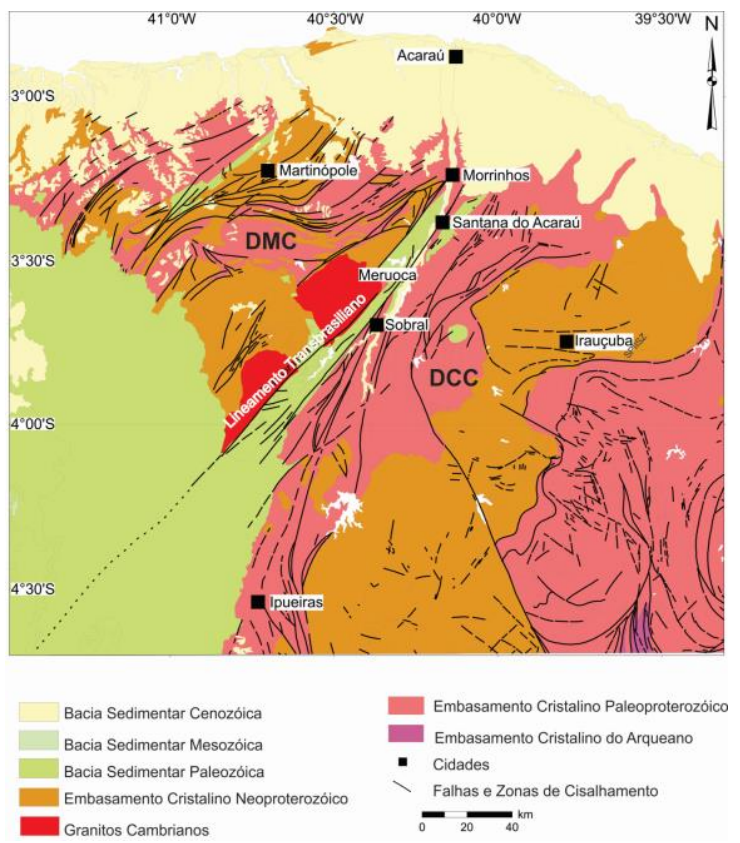

Figura 02 - Mapa geológico da área de estudo. DMC Domínio Médio Coreaú e DCC - Domínio Ceará Central. Carta Geológica do Brasil ao Milionésimo - Programa Geologia do Brasil, CPRM, Brasília. 2004.

\section{Metodologia}

Os sismos analisados foram registrados, no mínimo, em três estações de cada rede sismográfica local. A leitura dos tempos de chegada e das polaridades das ondas $\mathrm{Pe}$ $\mathrm{S}$, foram realizadas com o zuxílio do Programa SAC Seismic Analysis Code (Tapley \& Tull, 1991). Os epicentros, hipocentros e o modelo de velocidade foram determinados com o programa HYPO71 (Lee \& Lahr, 1975). Para determinar os parâmetros do modelo de crosta utilizado, inicialmente foi necessário encontrar um conjunto confiável de dados utilizando o diagrama de Wadati (Kissingler \& Engdahl, 1973), de forma conjunta. Sobre tais parâmetros, adotamos que a velocidade da onda $\mathbf{P}\left(\mathbf{V}_{\mathbf{P}}\right)$ e a razão $\mathbf{k}=\mathbf{V}_{\mathbf{p}} / \mathbf{V}_{\mathbf{S}}$ são consideradas constantes. As relocações hipocentrais foram realizadas com auxílio do programa HYPODD, com o objetivo de melhorar a precisão na localização hipocentral (Waldhauser \& Ellsworth, 2000) e para os mecanismos focais, foi utilizado o programa FPFIT (Reasemberg \& Oppenheimer 1985), considerando o primeiro movimento da onda P. Foram utilizadas somente as polaridades das ondas P com $100 \%$ de confiança e bem distribuídas (qualidade C1, como classificado por Assumpção et al., 2016). Como as estações sismográficas estão instaladas sobre embasamento Pré - Cambriano, constituído de rochas consolidadas e de baixa atenuação, observamos as chegadas das ondas $\mathrm{P}$ e $\mathrm{S}$ bem definidas nos sismogramas (Figura 03).

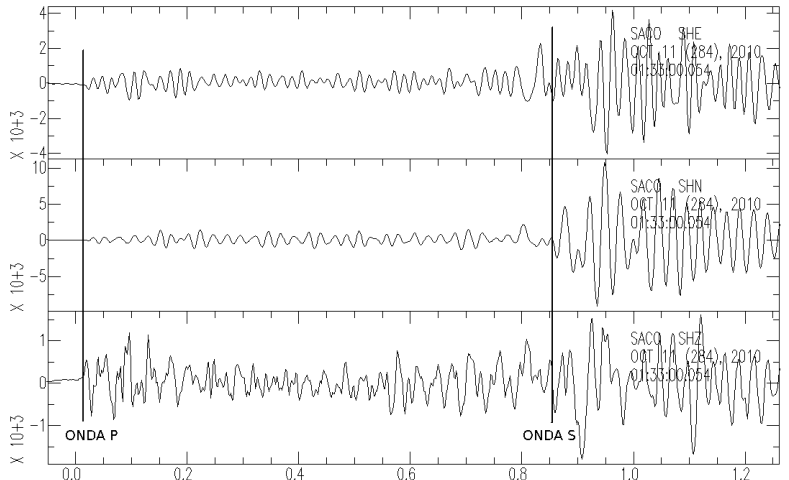

Figura 03 - Sismograma de um evento registrado na $Z S A$, mostrando as chegadas das ondas $P$ e $S$ bem claras. SHZ é a componente vertical, SHN a componente norte/sul e SHE a componente leste/oeste.

\section{Redes sismográficas}

\section{Local 1}

Nesta rede foram utilizados equipamentos do Laboratório de Estudos Litosféricos da UnB e estações de período curto do Projeto do Milênio e foi constituída de equipamentos fabricados pela Geotech Instruments, LCC, com estações providas de sismômetros de período curto (short period, SP), modelo S13J (com componentes: vertical, N-S e E-W), e de sismômetros de banda larga (broadband, BB), modelo KS2000M, tri-axial. Os sinais foram digitalizados pelo registrador SMART-24 (R) com três canais e 24bits.

\section{Local 2}

Esta rede foi composta por 6 estações digitais, com equipamentos pertencentes ao Pool de Equipamentos Geofísicos do Brasil (PEGBr), sediado no Observatório Nacional e com financiamento da PETROBRAS. Cada estação era composta por um sensor triaxial de período curto L4A (Sercel) e um registrador DAS 130 (Reftek). Esta rede operou entre dezembro de 2009 e dezembro de 2010 e registrou arquivos em contínuo, com taxa de amostragem de 100 amostras/s, com cada registro possuindo uma hora de duração.

\section{Local 3}

A rede composta neste local foi composta de sensores S$13 \mathrm{com}$ frequência natural de $1 \mathrm{hz}$, com registrador SMART 24 de 3 canais e 24 bits e operou entre setembro de 2015 e março de 2016, registrando com taxa de amostragem de 500 amostras/s (Menezes, 2017).

\section{Resultados e Discussão}

Para assegurar qualidade nos resultados e na determinação do modelo de velocidades local, foi escolhido dados consistentes de leituras das ondas $\mathrm{P}$ e $\mathrm{S}$ utilizando o diagrama de Wadati. Os modelos de velocidades obtidos foram: Local 1 , com $V p=6,0 \mathrm{~km} / \mathrm{s}$ e $V_{P} / V_{S}=1,709( \pm 0,001)$; Local $2 \mathrm{com} V p=6,0 \mathrm{~km} / \mathrm{s}$ e $V_{P} / V_{S}$ 
$=1,701( \pm 0,004)$ e Local 3 com $\mathrm{Vp}=6,2 \mathrm{~km} / \mathrm{s}$ e $\mathrm{V}_{\mathrm{p}} / \mathrm{V}_{\mathrm{S}}=$ $1,691( \pm 0,005)$, conforme Menezes, 2017. Os sismos na ZSA estão confinados na crosta superior e raramente ultrapassam profundidades hipocentrais maiores que 10 $\mathrm{km}$. Nos Locais 1 e 2, duas falhas sismogênicas foram identificadas: a Falha Riacho Fundo (FRF) e a Falha Santana (FS). Essas falhas surgiram através da liberação de esforços tectônicos orientados na direção NW-SE (SHmax) e na direção NE-SW (SHmin) e estão nas proximidades de uma importante feição geológica: o Lineamento Transbrasiliano e teve predominância de mecanismos com falhamentos trancorrentes, sendo que este é o tipo de falhamento que predomina no Nordeste do Brasil (Ferreira et al., 1998). No Local 3, os hipocentros mais rasos estão a $8 \mathrm{~km}$, os mais profundos a $9 \mathrm{~km}$ e o mecanismo focal mostrou falhamento normal (Menezes, 2017). A orientação da Falha Riacho coincide com as anomalias magnéticas no granito Meruoca e em seu embasamento (Moura et al., 2014). Há poucos casos como esse no mundo, como exemplo, na falha Hosgri, na costa central da California (Hardbeck, 2010) e na sismicidade de Caraíbas-Itacambi, em Minas Gerais (Chimpliganond et al., 2010). Este tipo de relação entre sismicidade intraplaca e anomalias magnéticas podem representar um indicador da possibilidade de ocorrência de atividade sísmica (Oliveira et al., 2015). Oliveira et al., 2015 calculou o esforço compressional máximo $\left(\sigma_{1}\right)$ para a ZSA e mostrou que ele está orientado na direção NWSE e o esforço compressional mínimo $\left(\sigma_{3}\right)$ está na direção NE-SW, utilizando os mecanismos focais encontrados nos Locais 1 e 2 . Esses resultados mostram como está o regime de esforços atual na Zona Sísmica Acaraú com $\sigma_{1}$ orientado paralelamente à margem passiva e $\sigma_{3}$ perpendicular à costa na parte noroeste da Província Borborema. Segundo Oliveira et al., 2015, os esforços nessas direções estão associados à superposição do esforço tectônico regional (devido à compressão da cordilheira meso-oceânica e dos Andes) com esforços tectônicos locais (devido à margem continental passiva). É necessário calcular os novos parâmetros do esforço tectônico com o mecanismo focal encontrado para o Local 3.

\section{Conclusões}

Através das análises detalhadas dos melhores eventos localizados na ZSA nos últimos dez anos, foi possível indentificar falhas sismogênicas ativas. A sismicidade tem uma considerável proximidade do Lineamento Transbrasiliano, está confinada na crosta superior e raramente ultrapassa profundidades hipocentrais maiores que $10 \mathrm{~km}$. Apesar dessa proximidade, não podemos afirmar que a sismicadade da ZSA nos últimos 10 anos, ocorreu devido à uma possível reativação do Lineamento Transbrasiliano. É muito difícil encontrar correlação entre sismicidade e reativações de zonas de cisalhamento, especialmente com o Lineamento Transbrasiliano (Oliveira et al., (2010); Moura et al., (2014)). Situação diferente é observada nas proximidades do Lineamento Pernambuco, pois Ferreira et al., 2008, Lima Neto et al., 2013 e Lopes et al., 2010 mostraram que a sismicidade ocorrida foi gerada pela a reativação dessa estrutura.

\section{Agradecimentos}

Os autores agradecem aos técnicos do LabSls pela instalação, operação e manutenção da rede de estações localizadas na Zona Sísmica Acaraú, ao INCT-ET pelos recursos financeiros e ao $\mathrm{PEGBr}$ pelos equipamentos de última geração utilizados.

\section{Referências}

ASSUMPÇÃO, M., DIAS, F.L., ZEVALLOS, I \& NALIBOFF, J.B., 2016. Intraplate stress field in South America from earthquake focal mechanisms, J. South. Am. Earth., 71, 278-295.

BEZERRA, F.H.R., do NASCIMENTO, A.F., FERREIRA, J.M., NOGUEIRA, F.C., FUCK, R.A., BRITO NEVES, B.B., SOUZA, M.O.L., 2011. Review of active faults in the Borborema Province, Intraplate South America Integration of seismological and paleoseismological data, Tectonophysics.

CABY, R. \& ARTHAUD, M.H., 1986. Major Precambrian nappes of the Brazilian Belts, Ceará, Northeast Brazil. Geol., 14, 871-874.

CPRM (Brazilian Geological Survey), 2009. Relatório final do levantamento e processamento dos dados magnetométricos e gamaespectrométricos. Projeto Aerogeofísico Norte do Ceará. Programa Geologia do Brasil (PGB). MME, SGM, 1, pp. 300.

CHIMPLIGANOND, C., ASSUMPÇÃO, M., VON HUELSEN, M. \& FRANÇAS, G. S., 2010. The intracratonic Caraíbas-Itacambi earthquake of December 09, $2010(4.9 \mathrm{mb})$, Minas gerais state, Brazil, Tectonophys., 480, 48-56.

FERREIRA, J.M. \& ASSUMPÇÃO, M., 1983. Sismicidade do Nordeste do Brasil, Rev. Bras. Geof., 1, 67-88.

FERREIRA, J.M., OLIVEIRA, R.T., TAKEYA, M.K. \& ASSUMPÇÃO, M., 1998. Superposition of local and regional stress in northeast Brazil: evidence from focal mechanism around the Potiguar marginal basin. Geophys. J. Int., 134: 341-355.

HARDEBECK, J.L., 2010. Seismotectonic and Fault Structure of the California Central Coast. Bull. Seismol. Soc. Am., 100, 1031-1050.

KISSSLINGER, C. \& ENGDAHL, E.R., 1973. The interpretation of the Wadati diagram with relaxed assumptions. Bull.Seism.Soc.Am. 63: 1723-1736.

LEE, W.H.K. \& LAHR, J.C., 1975. HYPO71 (revised): a computer program for determining hypocenter, magnitude and first motion pattern of local earthquakes. U.S. Geol. Surv. Open File Rep. 75-311, 114 pp.

LEE, W.H.K, STEWART, S.W., 1981. Principles and Aplications of Microearthquake Networks. Academic Press, Inc (London), LTD.

LIMA NETO, H.C., FERREIRA, J.M., BEZERRA., F.H.R., ASSUMPÇÃO, M., DO NASCIMENTO, A.F., SOUSA, 
M.O.L. \& MENEZES, E.A.S., 2013. Upper crustal earthquake swarms in São Caetano: Reactivation of the Pernambuco shear zone and trending branches in intraplate Brazil, Tectonophys., 633, 211-220.

LIMA NETO, H.C., FERREIRA, J.M., BEZERRA., F.H.R., ASSUMPÇÃO, M., DO NASCIMENTO, A.F., SOUSA, M.O.L. \& MENEZES, E.A.S., 2014. Earthquake sequences in the southern block of the Pernambuco Lineament, NE Brazil: Stress field and seismotectonic implications, Tectonophys.,608, 804-811.

LOPES, A.E.V., ASSUMPÇÃO, M., DO NASCIMENTO, A.F., FERREIRA, J.M., MENEZES, E.A.S. \& BARBOSA, J.R., 2010. Intraplate earthquake swarm in Belo Jardim, NE Brazil: reactivation of a major Neoproterozoic shear zone (Pernambuco Lineament), Geophy. J. Int., 180, 1303-1312.

MENEZES, E.A.S., 2017. Estudo da atividade sísmica em Irauçuba - CE entre setembro de 2015 a março de 2016. Dissertação de mestrado. PPGG/UFRN. 76 p.

MOURA, A.C.A., OLIVEIRA, P.H.S., FERREIRA, J.M., BEZERRA, F.H.R., FUCK, R.A. \& DO NASCIMENTO, A.F., 2014. Seismogenic faulting in the Meruoca granite, NE Brazil, consistent with a local weak fracture zone, Annals of the Braz. Acad. of Sci., 86 (4), 1631-1639.

OLIVEIRA, P.H.S., FERREIRA, J.M., do NASCIMENTO, A.F., BEZERRA, F.H.R., SOARES, J.E., FUCK, R.A., 2010. Estudo da Sismicidade na Região de Sobral - CE, NE do Brasil, em 2008. IV Simpósio da Sociedade Brasileira de Geofísica. Brasília - DF.

OLIVEIRA, P.H.S., FERREIRA, J.M., BEZERRA, F.H.R., ASSUMPÇÃO, M., DO NASCIMENTO, A.F., SOUSA, M.O.L. \& MENEZES, E.A.S., 2015. Influence of the continental margin on the stress field and seismicity in the intraplate Acaraú Seismic Zone, NE Brazil. Geophys. J. Int., 2015, 202 (3): 25-34.

REASENBERG, P. \& D. OPPENHEIMER., 1985. FPFIT, FPPLOT and FPPAGE: Fortran computer programs for calculating and displaying earthquake fault-plane solutions, U. S. Geol. Surv., 85-739.

SANTOS, T.J.S., FETTER, A.H, HACKSPACHER, P.C., NOGUEIRA NETO, J.A., 2008. Neoproterozoic Tectonic and Magmatic Episodes in the NW Sector of Borborema Province, NE Brazil, During Assembly of Western Gondwana. Journal of South American Earth Sciences. 25: 271-284.

TAPLEY W.C. \& TULL. J.E., 1991. SAC-Seismic Analysis Code, USER MANUAL. Livermore National Laboratory, $413 \mathrm{pp}$.

WALDHAUSER, F., ELLSWORTH, W.L., 2000. A Double - Difference Earthquakes Location Algorithm: Method and Application to the Northern Hayward Fault, California. Bulletim of the Seismological Society of America. v.90, n.6, pp.1353-1368. 